\title{
A Single Universal Force Field Can Uniquely Orient Non-Symmetric Parts
}

\author{
K.-F. Böhringer ${ }^{1}$, B.R. Donald ${ }^{2}$, L.E. Kavraki ${ }^{3}$ and F. Lamiraux ${ }^{3}$ \\ ${ }^{1}$ Department of Electrical Engineering, University of Washington, Seattle, WA 98195-2500, karl@ee.washington.edu \\ ${ }^{2}$ Department of Computer Science, Dartmouth College, Hanover, NH 03755-3510, brd@ cs.dartmouth.edu \\ ${ }^{3}$ Department of Computer Science, Rice University, Houston, TX 77005, \{kavraki|lamiraux $\} @$ cs.rice.edu
}

\begin{abstract}
Recent work in parts handling advocates the investigation of a new generation of devices for parts feeding, sorting, positioning, and assembly. Unlike robot grippers, conveyor belts, or vibratory bowl feeders, these devices generate force fields in which the parts move until they may reach a stable equilibrium pose.

The development of the theory of programmable force fields has yielded a number of strategies to uniquely position and orient parts. Typically, more than one fields are applied in sequence to achieve the desired result. In this paper we show that unique part poses can be achieved with a single field. In particular, we present a single field that positions and orients any non-symmetric part into two stable equilibrium poses. Then we show that for any laminar part there exists a field in which the non-symmetric part reaches a unique stable equilibrium pose. Our latter result leads to the design of devices that can act as "universal parts feeders" proving an earlier conjecture about their existence.
\end{abstract}

\section{Introduction}

Part manipulation is an important but also timeconsuming operation in industrial automation. Traditionally part feeding and orienting has been performed with vibratory bowl feeders. These devices are customly designed for the orientation of a single part or a small number of parts and rely on mechanical fi lters to reject parts in unwanted orientations. Despite their widespread use, vibratory bowl feeders have several disadvantages: they have to be redesigned when the geometry of the part changes; they may damage parts that repeatedly run through the mechanical fi lters, etc.

Recent work investigates alternative ways for feeding parts in assembly workcells. Parts feeders that are programmed, rather than mechanically modifi ed, offer an attractive solution since they can be used for a variety of parts [1, 2, 3, 4]. Practical considerations favor feeding methods that require little or no sensing, em-

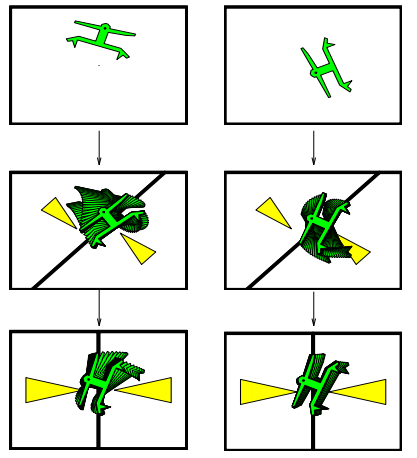

Figure 1: Sensorless parts orienting using a sequence of squeeze fields: The arrows indicate the direction of the force field.

ploy simple devices, and are as robust as possible. One of the proposed alternatives is the use of programmable force fi elds $[2,5,6]$. The basic idea is the following: the fi eld is realized on a planar surface on which the part is placed. The forces exerted on the contact surface of the part translate and rotate the part to an equilibrium pose. The manipulation requires no sensing.

Current technology permits the implementation of certain force fields in the microscale with actuator arrays [5] built in micro electro mechanical system (MEMS) technology, and in the macroscale with transversely vibrating plates. The flexibility and dexterity that programmable force fi elds offer has led researchers to investigate the extent to which these fi elds can be useful. The work in [5] analyzes the properties of force fi elds that are suitable for sensorless manipulation and proposes novel manipulation strategies. These strategies typically consist of sequences of force fi elds that cascade the parts through multiple equilibria until a desired goal state is reached. Figure 1 shows such a two-step sequence to orient a polygonal part (Reprinted from [2]).

Programmable force fi elds allow us to shift the complexity of parts feeding from the design of mechan- 
ical tracks, and fi lters to control algorithms and circuitry. No sensors or feeder re-design is required. However, the designs proposed in [5] require control software, a clock, and, to some extent, synchronization between distributed actuators. In this paper we show that the device complexity can be further reduced, by presenting two specifi c device architectures. Assuming non-symmetric parts, the fi rst design achieves exactly two stable equilibria without sensor feedback, clock, or control system. More precisely, unique positioning and orienting is reached modulo $180^{\circ}$ in orientation. The second design overcomes this limitation and for any non-symmetric part achieves unique positioning and orientation. We explain that our second result demonstrates the first known instance of a universal feeder/orienter (UFO) device [2], i.e., a general purpose device that can uniquely position and orient any part without redesigning or reprogramming.

\section{Related Work}

An extensive survey of previous and related work in the area of programmable force fi elds is offered in [7]. In this paper we only give a brief summary.

In 1994 Böhringer and Donald proposed that there exist challenging algorithmic problems in MEMS and programmable force fi elds, at the intersection of combinatorial algorithms, geometry, dynamical systems, and distributed systems [5]. From 1993-1998, Böhringer and Donald worked with Noel MacDonald at the Cornell Nanofabrication Facility to develop and test new arrays of MEMS microactuators for programmable force fi elds [2, 5, 8]. At the same time, Böhringer and Donald worked with Greg Kovacs' group at the Center for Integrated Systems at Stanford, to develop a control system for MEMS organic ciliary arrays, and to perform experiments with these arrays to manipulate IC dice using array-induced force fi elds [9]. In parallel, Böhringer and Donald worked with Ken Goldberg at Berkeley and Vivek Bhatt at Cornell to generalize the theory to macroscopic devices, by developing algorithms for transversely vibrating plates in order to implement programmable force fi elds [10]. Around this time, Lydia Kavraki explored the power of continuous force fi elds, and demonstrated an elliptical potential fi eld capable of posing any part into one of two equilibrium states [11], and investigated the effect of control uncertainty on the stability of equilibria. Finally, Böhringer and Donald worked with Dan Halperin, to develop new upper and lower bounds and a precise analysis of the area bisectors arising in squeeze-fi eld algorithms [12].

Until recently, work on force fi elds for manipulation has been dominated by the artifi cial potential fi elds pioneered by Khatib, Koditschek, and Brooks. While potential fi elds have been widely used in robot control [13, 14], micro-actuator arrays present us with the ability to explicitly program the applied force at every point in a force fi eld. Several groups have described efforts to apply MEMS actuators to positioning, inspection, and assembly tasks with small parts $[5,6,15,16]$. However, the fabrication, control, and programming of micro-devices that can interact and actively change their environment remains challenging.

Other groups have also been active in developing new devices, analysis, and algorithms. John Canny and Dan Reznik developed sophisticated dynamic models and dynamic simulators for both MEMS devices and macroscopic vibrating plates [17]. Peter Will and his colleagues at USC-ISI have explored a number of different MEMS array designs, as well as algorithms and analysis for programmable force fi elds $[6,18]$. Andy Berlin, David Biegelsen, and Warren Jackson at Xerox PARC have developed a novel MEMS microactuator array based on controllable air jets, with integrated control and sensing circuitry [19]. Working at CMU, Bill Messner and Jonathan Luntz developed a small room whose floor is tiled with controllable, programmable, macroscopic wheels that can be driven and steered to manipulate large objects such as boxes [20]. Their system employed distributed, local controllers to implement programmable force fi elds. Together with Howie Choset, they analyzed the resulting dynamical system to obtain interesting results on controllability and programmable force fi eld algorithms based on conservative vs. non-conservative fi elds [21]. Working with the Berkeley Sensor \& Actuator Center (BSAC), Karl Böhringer and Ken Goldberg explored how MEMS devices employing electrostatic fringing fi elds can be used to implement programmable force fi elds for parts manipulation and self-assembly [22].

In short, there has been an explosion of new and exotic arrayed devices for both MEMS manipulation and macroscopic manipulation. Despite advances, however, the conjecture [2] about the existence of a Universal Feeder-Orienter (UFO) Device has remained open since 1995; the problem has been widely viewed as resistant to solution. In this paper, we prove the conjecture is true.

\section{Force Fields for Part Manipulation}

In this section we summarize some of the basic results in the theory of programmable force fi elds that are necessary for the remainder of the paper. In a programmable force fi eld, every point in the plane is 
associated with a force vector in the plane. For example, a unit squeeze fi eld is defi ned as $\mathbf{f}(x, y)=$ $-\operatorname{sign}(x)(1,0)$. When a part is placed into a squeeze fi eld, it experiences a translation and re-orientation until a predictable equilibrium is reached. This property makes squeeze fi elds very useful for sensorless positioning and orienting strategies.

Given a polygonal part $P$ with $n$ vertices, it was shown in [5] that there exist $O\left(n^{2} k\right)$ stable equilibrium orientations for $P$ when placed in $\mathbf{f}$ ( $k$ is the number of combinatorially distinct bisector placements for $P$ [12]. This result was used to generate strategies for unique parts posing (up to symmetry) by reducing the problem to a parts feeding algorithm developed by Goldberg [4]. The strategies have length $O\left(n^{2} k\right)$ and can be generated in $O\left(n^{4} k^{2}\right)$ time.

In [2] this result was improved to plan lengths of $O(n k)$ and planning time $O\left(n^{2} k^{2}\right)$, by employing combined squeeze and unit radial fi elds (unit radial fi elds are defi ned as $\mathbf{r}(x, y)=\left(-1 / \sqrt{x^{2}+y^{2}}\right)(x, y)$ and are described in more detail in Section 6.).

Using elliptic force fi elds $\mathbf{f}(x, y)=(-\alpha x,-\beta y)$ such that $0<\alpha<\beta$, this bound can be reduced to a constant number (2) independent of $n$ [11]. We show this result in Section 5..

It was conjectured in [2] that a fi eld which combines a radial and gravitational fi eld $\mathbf{r}+\delta \mathrm{g}(\mathrm{g}(x, y)=$ $(0,-1)$ and $\delta$ is a small positive constant), has the property of uniquely orienting and positioning parts. We call this fi eld the radial-gravity fi eld and we prove in Section 6. that for any non-symmetric part, there is a radial-gravity fi eld inducing exactly one stable equilibrium. Our paper also includes a discussion on implementation issues relating to the radial-gravity fi eld. Such a fi eld could be used to build a universal parts feeder (inspired by the "universal gripper" as proposed by Abell and Erdmann [23] ${ }^{1}$ ). In contrast to the universal manipulator fi elds proposed in [17], such a device could uniquely position a part without the need of a clock, sensors, or programming.

\section{Conditions for Equilibria}

In this section we give some defi nitions and establish the notation that will be used in the two following sections. We investigate the conditions for equilibrium for a part $w$ in the presence of a force fi eld $\mathbf{f}: \mathbb{R}^{2} \rightarrow \mathbb{R}^{2}$. It is assumed that $w(x, y) \geq 0$, for $x, y \in \mathbb{R}$, and

\footnotetext{
${ }^{1}$ In a universal gripper a part is free to rotate after being picked up from an arbitrary initial state. Its center of mass will settle at the unique minimum of potential energy, causing the part to reach a unique, predictable equilibrium.
}

$W=\int_{\mathbb{R}^{2}} w(\mathbf{p}) d \mathbf{p}<\infty$. Here $w$ can be seen as the support (characteristic) function of the part, this function is 1 on the part and 0 elsewhere. We assume that the support of $w$ is compact.

Without loss of generality, the origin of the reference frame in the plane can be chosen as the center of mass of $w$ :

$$
\int_{\mathbb{R}^{2}} \mathbf{p} w(\mathbf{p}) d \mathbf{p}=\mathbf{0} .
$$

When the part is in confi guration $q=(x, y, \theta)$, the resultant force is given by

$$
\mathbf{F}=\int_{\mathbb{R}^{2}} w(\mathbf{p}) \mathbf{f}\left(A_{\theta} \mathbf{p}+\mathbf{t}\right) d \mathbf{p},
$$

and the resultant torque at the center of mass is given by

$$
\mathbf{M}=\int_{\mathbb{R}^{2}} w(\mathbf{p})\left(A_{\theta} \mathbf{p}\right) \times \mathbf{f}\left(A_{\theta} \mathbf{p}+\mathbf{t}\right) d \mathbf{p},
$$

where $\mathbf{t}=(x, y)^{\top}$, and is the rotation matrix of angle $\theta$. From now on, all integrals extend over $\mathbb{R}^{2}$ unless otherwise stated.

A total equilibrium is achieved when the resultant force and torque on the part is zero. For a total equilibrium the following two equations must hold:

$$
\begin{aligned}
\mathbf{F} & =\mathbf{0} \\
\mathbf{M} & =\mathbf{0} .
\end{aligned}
$$

\section{Two Stable Equilibrium Orientations}

In this section we show a force fi eld that orients most parts into two stable equilibria. The fi eld derives from an elliptic potential fi eld and we call it the elliptic fi eld:

$$
\mathbf{f}(x, y)=(-\alpha x,-\beta y)
$$

where $\alpha$ and $\beta$ are two distinct positive constants. Without loss of generality let us assume that $\alpha<\beta$. Figure 2 displays one such force fi eld with $\alpha=1$ and $\beta=2$. Note that this force fi eld is the negative gradient of the elliptic potential function $u(x, y)=\frac{\alpha}{2} x^{2}+\frac{\beta}{2} y^{2}$. This potential function is plotted in Figure 3, for $\alpha=1$ and $\beta=2$.

\subsection{Force Equilibrium}

We fi rst establish the condition for the force equilibrium. If $(x, y)$ are the coordinates of the center of mass of $w$ in confi guration $\mathbf{q}$, ( $\mathrm{W}$ is defi ned in Section 4.), the total force exerted on $w$, is equal to

$$
(-\alpha W x,-\beta W y) \text {. }
$$




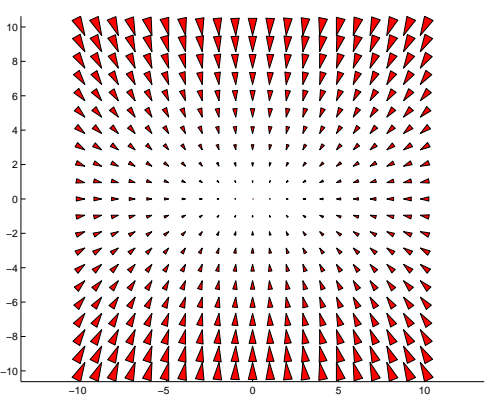

Figure 2: Force field for $\alpha=1$ and $\beta=2$.

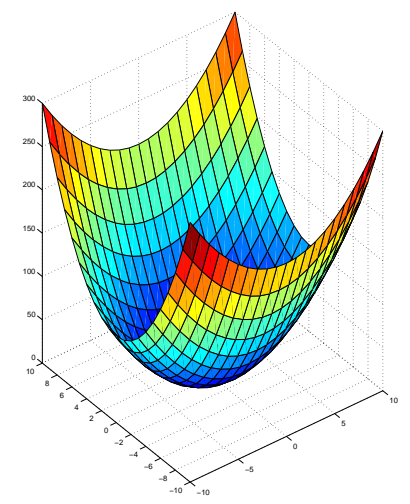

Figure 3: Elliptic potential for $\alpha=1$ and $\beta=2$.

Condition (1) is thus equivalent to $(x, y)=(0,0)$. Therefore, for the equilibrium confi gurations $\mathbf{q}$, we only need to consider confi gurations of the type $\mathbf{q}=$ $(0,0, \theta)$.

\subsection{Moment Equilibrium}

We now proceed to the investigation of condition (2). It turns out that, for "most" parts $w$ and for whatever distinct positive values of $\alpha$ and $\beta$, there are exactly 4 values of $\theta$ for which (2) holds. This is shown below.

Taking into account the force equilibrium, the expression of the torque becomes now

$$
\mathbf{M}=\int w(\mathbf{p})\left(A_{\theta} \mathbf{p}\right) \times \mathbf{f}\left(A_{\theta} \mathbf{p}\right) d \mathbf{p} .
$$

After calculations

$$
\mathbf{M}=(\alpha-\beta)\left(\cos 2 \theta \int x y w(x, y) d x d y\right) \cdot \mathbf{k} .
$$

In the above

$$
s_{m n}=s_{m n}(w)=\int_{\mathbb{R}^{2}} x^{m} y^{n} w(x, y) d x d y
$$

define moments of $w$. Thus, since $\alpha \neq \beta$, we have $\mathbf{M}=\mathbf{0}$ if and only if $\frac{s_{20}-s_{02}}{2} \sin 2 \theta+$ $s_{11} \cos 2 \theta=0$. Equivalently, we want the vectors $(\cos 2 \theta, \sin 2 \theta)$ and $\left(s_{11}, \frac{1}{2}\left(s_{20}-s_{02}\right)\right)$ to be orthogonal. This leads to the following theorem:

Theorem 1 Let $w: \mathbb{R}^{2} \rightarrow \mathbb{R}$ be a part with finite $s_{i j}$ with $i+j \leq 2$ and whose "center of mass" is at $\mathbf{0}$, and let $\mathbf{f}(x, y)=(-\alpha x,-\beta y)$, with $0<\alpha<\beta$, be the underlying force field.

"SYMMETRY": If $s_{11}=s_{20}-s_{02}=0$ the part $w\left(A_{\theta} \mathbf{p}+\mathbf{t}\right)$ is at total equilibrium whenever $\mathbf{t}=\mathbf{0}$.

"Asymmetry": Otherwise, the distribution $w\left(A_{\theta} \mathbf{p}+\right.$ t) is in equilibrium only when $\mathbf{t}=0$ and for exactly 4 distinct values of $\theta \in[0,2 \pi)$. These 4 values of $\theta$ are $\frac{\pi}{2}$ apart and only 2 of them, say $\theta_{0}$ and $\theta_{0}+\pi$, represent stable equilibria, the others, $\theta_{0}+\frac{\pi}{2}$ and $\theta_{0}+\frac{3 \pi}{2}$ being unstable.

\section{One Stable Equilibrium Orientation}

We now exhibit a class of force fi elds that induce one stable equilibrium for most parts. These fi elds are combinations of a unit radial and gravity fi eld and we will call them radial-gravity fields:

- A unit radial field $R$ is defined by: $\mathbf{r}(x, y)=$ $-\frac{1}{\sqrt{x^{2}+y^{2}}}(x, y)$.

- A unit gravity field $\mathrm{g}$ is given by $\mathrm{g}(x, y)=$ $(0,-1)$.

- For a given $\delta \in \mathbb{R}$, the radial-gravity field is defi ned as the sum of a unit radial fi eld $\mathbf{r}$ and a gravity fi eld $\mathrm{g}$ scaled by $\delta: F_{\delta}=\mathbf{r}+\delta \mathrm{g}$.

Figures 4 and 5 plot a radial-gravity fi eld for which $\delta=0.4$.

Equilibrium through Potential Fields In this section we reason with potential fi elds instead of using directly equations ( 1 ) and (2). First we notice that $F_{\delta}$ derives from the potential fi eld $u_{\delta}(x, y)=\sqrt{x^{2}+y^{2}}-$ $\delta y$ and we defi ne the following potential fi eld over the confi guration space $\mathcal{C}$ of the part:

$$
U_{\delta}(q)=\int w(\mathbf{p}) u_{\delta}\left(A_{\theta} \mathbf{p}+\mathbf{t}\right) d \mathbf{p} .
$$

A confi guration $q$ is a stable equilibrium of the part if and only if $q$ is a local minimum of the function $U_{\delta}$.

In order to take advantage of the radial symmetry of $\mathbf{r}(x, y)$, we defi ne a new system of coordinates $(X, Y, \theta)$ from the standard one by

$$
\begin{aligned}
& X=x \cos \theta+y \sin \theta \\
& Y=-x \sin \theta+y \cos \theta .
\end{aligned}
$$




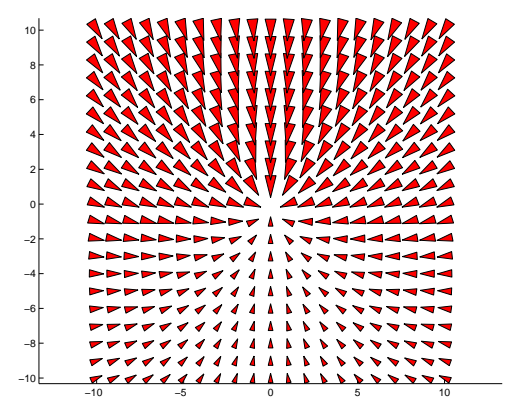

Figure 4: Radial-gravity field with $\delta=0.4$.

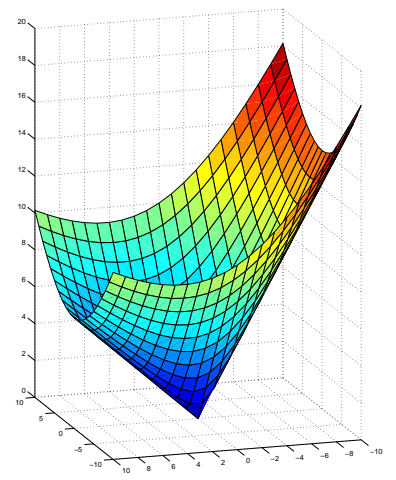

Figure 5: Combination of a unit radial and a gravitational potential field with $\delta=0.4$.

The expression of $U_{\delta}$ in this new system of coordinates is obtained by a change of variable in the integral:

$$
\begin{aligned}
& U_{\delta}(X, Y, \theta)= \\
& \int w(\xi, \eta) \sqrt{(X+\xi)^{2}+(Y+\eta)^{2}} d \xi d \eta \\
& -\delta W(X \sin \theta+Y \cos \theta) .
\end{aligned}
$$

To establish the existence and uniqueness of a stable equilibrium, we proceed in two steps. First we state the existence and uniqueness of a local minimum of the potential fi eld for any fi xed $\theta$. This partial minimum is the force equilibrium. Then we study the curve of force equilibria when $\theta$ describes $\mathbf{S}^{1}$ and reason about moment equilibria. For our discussion below, we defi ne the following functions:

$$
U_{\theta, \delta}(X, Y)=U(X, Y, \theta, \delta)=U_{\delta}(X, Y, \theta) .
$$

\subsection{Force Equilibrium}

A force equilibrium is a local minimum of $U_{\theta, \delta}$. Using common results of the theory of integration, we find that $U$ is of the class $C^{2}$ and that its partial derivatives with respect to $X$ and $Y$ are obtained by differentiating under the integral. The following proposition establishes the existence and uniqueness of a stable force equilibrium for a fi xed $\delta$ by proving that the function $U_{\theta, \delta}$ is convex.

Proposition 2 If $\delta<1, U_{\theta, \delta}$ has a unique local minimum.

The proof of this proposition can be found in [7].

\subsection{Moment Equilibrium}

Having established the force equilibrium, we proceed to express it as a function of $\theta, \delta$.

Equilibrium Curve We denote the unique force equilibrium relative to $\theta$ by $\left(X^{*}(\theta, \delta), Y^{*}(\theta, \delta)\right)$ and by $\left(x^{*}(\theta, \delta), y^{*}(\theta, \delta)\right)$ its expression in the $(x, y, \theta)$ system of coordinates:

$$
\begin{aligned}
& x^{*}(\theta, \delta)=\cos \theta X^{*}(\theta, \delta)-\sin \theta Y^{*}(\theta, \delta) \\
& y^{*}(\theta, \delta)=\sin \theta X^{*}(\theta, \delta)+\cos \theta Y^{*}(\theta, \delta)
\end{aligned}
$$

We call equilibrium curve of parameter $\delta$ the curve $\left\{\left(x^{*}(\theta, \delta), y^{*}(\theta, \delta)\right), \theta \in \mathbf{S}^{1}\right\}$ of force equilibria.

When $\delta=0$ (pure radial fi eld), due to the radial symmetry of the fi eld, the set of equilibrium confi gurations is generated by the rotations of the part about one of its points called the pivot point [5].

We have established in [7] that $X^{*}, Y^{*}, x^{*}, y^{*}$ are continuously differentiable. Let us now denote by $U_{\delta}^{*}(\theta)$ the minimum value of the potential function for each $\theta$. Then it is straightforward that $(X, Y, \theta)$ is a local minimum of $U_{\delta}$ if and only if $\theta$ is a local minimum of $U_{\delta}^{*}$ and $X=X^{*}(\theta, \delta)$ and $Y=Y^{*}(\theta, \delta)$. The following proposition establishes a relation between the derivative of $U_{\delta}^{*}$ and the position in the plane of the force equilibrium.

Proposition $\mathbf{3}$ For any $\theta \in \mathbf{S}^{1}$,

$$
\frac{d U_{\delta}^{*}}{d \theta}(\theta)=\delta W x^{*}(\theta, \delta) .
$$

The proof of this proposition can be found in [7].

Proposition 3 states that a stable equilibrium confi guration corresponds to a value of $\theta$ where the equilibrium curve crosses the $\mathrm{y}$-axis from $x<0$ to $x>0$. We now proceed to establish the unique global equilibrium.

Theorem 4 (Unique Global Equilibrium) For any compact part $w$, if $\left(X^{*}(\theta, 0), Y^{*}(\theta, 0)\right) \neq(0,0)$ (i.e. 


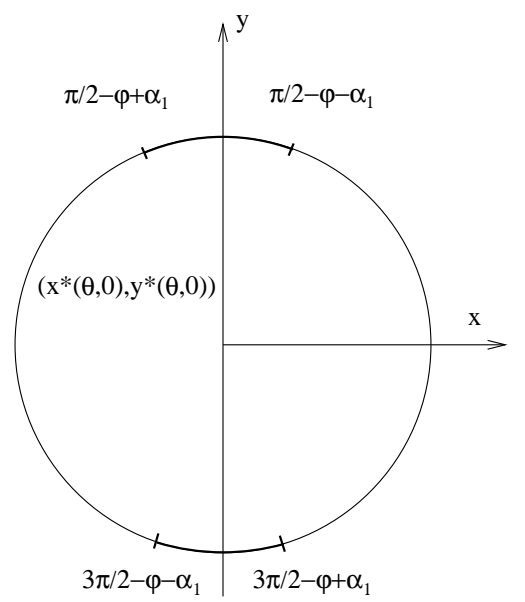

Figure 6: Decomposition of the equilibrium curve for $\delta=0$ into four intervals.

the center of mass and the pivot point are distinct) then there exists $\delta>0$ such that $w$ has a unique stable equilibrium configuration under the potential field $U_{\delta}$.

Proof: First, let us notice that the curve $\left(X^{*}(\theta, 0), Y^{*}(\theta, 0)\right)$ is reduced to a point since when $\delta=0$, the potential fi eld $U_{\delta}$ does not depend on $\theta$. Let us express this point in polar coordinates

$$
\begin{aligned}
& X^{*}(\theta, 0)=R \cos \varphi \\
& Y^{*}(\theta, 0)=R \sin \varphi .
\end{aligned}
$$

Then if $\left(X^{*}, Y^{*}\right) \neq(0,0)$, from relations (6) and (7), the curve $\left(x^{*}(\theta, 0), y^{*}(\theta, 0)\right)$ is a circle centered on $(0,0)$ (Figure 6).

$$
\begin{aligned}
& x^{*}(\theta, 0)=R \cos (\theta+\varphi) \\
& y^{*}(\theta, 0)=R \sin (\theta+\varphi) .
\end{aligned}
$$

The current proof is based on the continuity of the functions $x^{*}$ and $y^{*}$ and their derivatives. We proceed in two steps: near $\pi / 2-\varphi$ and $3 \pi / 2-\varphi$, where $x^{*}(\theta, 0)$ crosses 0 , the variation of the tangent vector to the curve $\left(x^{*}(\theta, \delta), y^{*}(\theta, \delta)\right)$ can be made suffi ciently small in order to prevent the curve to cross twice the $y$-axis. For the remaining values of $\theta$, the variation of the position of the curve can be bounded in such a way that the curve cannot cross the y-axis. The complete proof follows.

Let us recall that $\partial x^{*} / \partial \theta(\theta, \delta)$ is a continuous function and that $\partial x^{*} / \partial \theta(-\varphi+\pi / 2,0)=-R$ and $\partial x^{*} / \partial \theta(3 \pi / 2-\varphi, 0)=R$. Therefore there exists $\alpha_{1}>0$ and $\delta_{1}>0$ such that $\forall \delta<\delta_{1}, \forall \theta \in$ $\left[-\varphi+\pi / 2-\alpha_{1},-\varphi+\pi / 2+\alpha_{1}\right], \frac{\partial x^{*}}{\partial \theta}(\theta, \delta)<0$ and $\forall \delta<\delta_{1}, \forall \theta \in\left[-\varphi+3 \pi / 2-\alpha_{1},-\varphi+3 \pi / 2+\right.$ $\left.\alpha_{1}\right], \frac{\partial x^{*}}{\partial \theta}(\theta, \delta)>0$.
These inequalities imply that the equilibrium curve does not cross more than once the y-axis on the corresponding intervals of $\theta$.

We are going now to show that for the remaining values of $\theta$, there exists a $\delta$ small enough such that the corresponding part of the equilibrium curve does not cross the y-axis. To make the notation clearer, let us defi ne the following compact set

$$
\begin{aligned}
I= & {\left[-\varphi+\pi / 2+\alpha_{1},-\varphi+3 \pi / 2-\alpha_{1}\right] \cup } \\
& {\left[-\varphi+3 \pi / 2+\alpha_{1},-\varphi+5 \pi / 2-\alpha_{1}\right] }
\end{aligned}
$$

Then for $\delta=0$ and $\theta \in I$, the equilibrium curve stays at a strictly positive distance from the y-axis:

$$
\alpha_{2}=\operatorname{Inf}\left\{\left|x^{*}(\theta, 0)\right|, \theta \in I\right\}>0 .
$$

$x^{*}$ is continuous, thus its restriction to the compact set $I \times\left[0, \delta_{1}\right]$ is uniformly continuous. Therefore, there exists a constant $\delta_{2}>0$ such that

$$
\forall \theta \in I, \forall \delta \in\left[0, \delta_{2}\right],\left|x^{*}(\theta, \delta)-x^{*}(\theta, 0)\right|<\alpha_{2} .
$$

This condition ensures that the equilibrium curves does not cross the y-axis for $\theta \in I$ and $\delta<\delta_{2}$.

Therefore, for any $\delta<\min \left(\delta_{1}, \delta_{2}\right)$, the equilibrium curve crosses the y-axis at most twice, once in each direction.

The previous computation shows that if a part has a pivot point different from the center of mass, then there exists a small value of $\delta$ to uniquely orient this part. However, this does not mean that there exists one unique value of $\delta$ orienting any part. In other words, the combination of a radial unit fi eld and a gravitational fi eld is a strategy that can orient almost any part, but for each part the maximum $\delta$ is different. For each part, the value of $\delta_{\max }$ can be computed numerically.

Figure 7 shows equilibrium curves for the ratchet for different values of $\delta$. In this example, we can see that for large $\delta$, the equilibrium curve crosses the $y-$ axis several times, and thus the minimum is not unique anymore.

\section{Simulation}

We have implemented a sophisticated simulator for programmable force fi elds in MATLAB. The system is capable of exact calculation of the force acting on polygonal parts in various fi elds, including squeeze, unit radial, gravity fi elds, and combinations thereof. To calculate the force acting on a polygon in the fi eld, the polygon is triangulated and the force fi eld is integrated over the individual areas. This is done without numerical integration since there exist closed-form integrals 

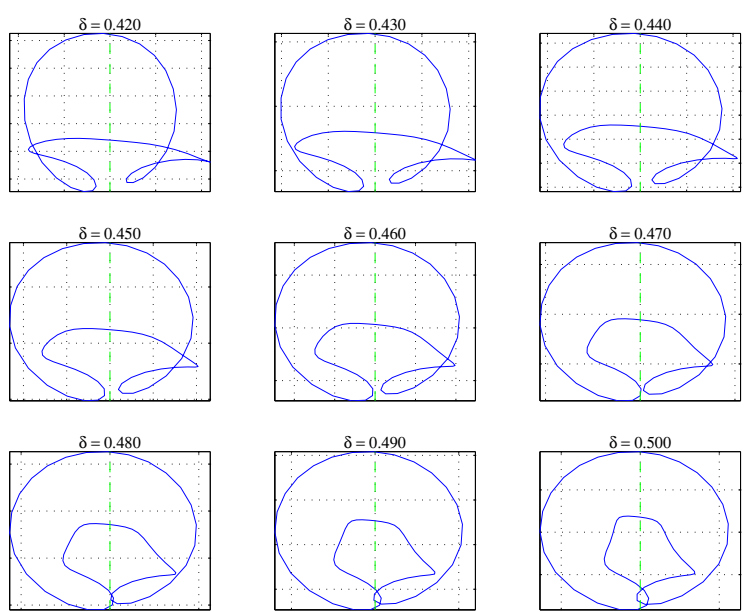

Figure 7: Detailed equilibrium curves for the ratchet: from $\delta=0.42$ to $\delta=0.50$, increment 0.01 . We observe that up to $\delta=0.46$ the curve has only two intersections with the $y$-axis, hence the equilibrium is unique.

for all these fi elds. To predict the part motion in the fi eld, we have implemented a full dynamic simulator that includes inertia, viscous damping, and Coulomb friction. Force equilibria are determined numerically by solving the constraints $\mathbf{F}=0$ as given in equation 1. Pivot points are also determined numerically.

\section{Device Construction}

In Section 2. we have already mentioned some device designs that implement programmable force fields. Several designs may be possible.

Elliptic Fields The realization of elliptic fi elds could be achieved with MEMS actuator arrays [8], or arrays of motors [21], and possibly with vibrating plates. The main challenge for vibrating plates will be to obtain a surface that approximates the elliptic force profi le with suffi cient spatial resolution. Microscopic (MEMS) or macroscopic (motor) actuator arrays offer alternatives $[15,16,5,6]$. The main challenge for micro actuators remains the generation and control of forces over a suffi ciently large range of force magnitudes.

Universal Fields A prototype unidirectional array was built by Böhringer et al. [8] (see Figure 8). This array can generate a unit gravity fi eld. Its design could be modifi ed such that the actuators are arranged in a circular pattern, which would result in a unit radial fi eld. The variable gravity fi eld could then be added simply by tilting the array accordingly (see Figure 9). Hence such a device would be relatively easy to build. The

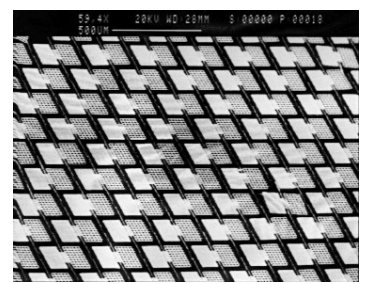

Figure 8: Unidirectional MEMS actuator array built on a silicon wafer. Each actuator is $\sim 0.2 \mathrm{~mm}$ in size.

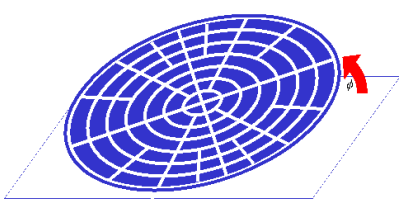

Figure 9: Conceptual design of an actuator array that implements a combined radial-gravity field. Individual actuators are tiled in a circular array pattern. The array is tilted between $\phi=0^{\circ}$ and $45^{\circ}$ to add a gravity component $\delta \mathrm{g}$ with $\delta=\tan \phi$.

key observation is that with current MEMS technology it is easy to build actuator arrays with high spatial resolution $(\ll 1 \mathrm{~mm})$ and constant force, but it is diffi cult to build actuators with variable force. In addition, MEMS actuators can be easily arranged into arbitrary patterns (in particular, a radial pattern). Hence it is easy to build arrays that implement unit radial fi elds. Alternatively, a resonating speaker, or a vibrating diskshaped plate that is fi xed at the center, might be used to create a radial force fi eld.

\section{Discussion}

This paper proves the existence of devices for parts positioning and orienting that can bring arbitrary (nonsymmetric) parts into exactly one or two stable equilibria. These devices are extremely simple: they do not require a feedback control, a clock, synchronization, or programming. Their functioning principle is based on force vector fi elds. Such a device could revolutionize industrial and precision parts handling.

Acknowledgments Work on this paper by Karl B ohringer and Bruce Randall Donald has been supported in part by the National Science Foundation under Grant Nos. IRI-8802390, IRI-9000532， IRI-9201699， IRI-9530785, IRI-9896020, NSF EIA-9901407, NSF IIS-9906790, NSF 9802068, NSF CDA-9726389, NSF EIA-9818299, 
NSF CISE/CDA-9805548, by an equipment grant from Microsoft Research, by a Presidential Young Investigator award to Bruce Donald, by an NSF/ARPA Small Grant for Exploratory Research No. IRI-9403903, by an NSF CISE Postdoctoral Associateship to Karl B ohringer No. CDA-9705022, and in part by the Air Force Office of Sponsored Research, the Mathematical Sciences Institute, Intel Corporation, and AT\&T Bell laboratories. Work on this paper by Lydia Kavraki and Florent Lamiraux has been supported in part by NSF IRI-970228 and NSF CISE SA1728-21122N. The authors would like to thank Eric Babson, Mike Erdmann, and Andy Ruina for valuable discussions.

\section{References}

[1] S. Akella, W. H. Huang, K. M. Lynch, and M. T. Mason. Planar manipulation on a conveyor by a one joint robot with and without sensing. In International Symposium of Robotics Research (ISRR), 1995.

[2] K.-F. B ohringer, B. R. Donald, and N. C. MacDonald. Programmable vector fields for distributed manipulation, with applications to MEMS actuator arrays and vibratory parts feeders. Int. Journal of Robotics Research, Feb. 1999.

[3] M. Erdmann. An exploration of nonprehensile twopalm manipulation: Planning and execution. In G. Giralt and G. Hirzinger, editors, Robotics Research, pages 16-27. Springer Verlag, 1996.

[4] K. Y. Goldberg. Orienting polygonal parts without sensing. Algorithmica, 10(2/3/4):201-225, August/September/October 1993.

[5] K.-F. B ohringer, B. R. Donald, R. Mihailovich, and N. C. MacDonald. Sensorless manipulation using massively parallel microfabricated actuator arrays. In Proc. IEEE Int. Conf. on Robotics and Automation (ICRA), pages 826-833, San Diego, CA, May 1994.

[6] W. Liu and P. Will. Parts manipulation on an intelligent motion surface. In IEEE/RSJ Int. Workshop on Intelligent Robots \& Systems (IROS), Pittsburgh, PA, 1995.

[7] K.-F. B ohringer, B. R. Donald, L. Kavraki, and F. Lamiraux. Part orientation with one or two stable equilibria using programmable vector fields. IEEE Transactions on Robotics and Automation, 2000. To appear.

[8] K.-F. B ohringer, B. R. Donald, and N. C. MacDonald. Single-crystal silicon actuator arrays for micro manipulation tasks. In Proc. IEEE Workshop on Micro Electro Mechanical Systems (MEMS), pages 7-12, San Diego, CA, Feb. 1996.

[9] J. W. Suh, R. B. Darling, K. F. B ohringer, H. Baltes, B. R. Donald, and G. T. A. Kovacs. CMOS integrated organic ciliary array for general-purpose micromanipulation tool for small objects. Journal of Microelectromechanical Systems, 1998. Submitted for review.
[10] K.-F. B ohringer, V. Bhatt, B. R. Donald, and K. Y. Goldberg. Sensorless manipulation using transverse vibrations of a plate. Algorithmica, 1999. Special Issue on Algorithmic Foundations of Robotics. Accepted for publication. Forthcoming.

[11] L. Kavraki. Part orientation with programmable vector fields: Two stable equilibria for most parts. In Proc. IEEE Int. Conf. on Robotics and Automation (ICRA), Albuquerque, New Mexico, Apr. 1997.

[12] K.-F. B ohringer, B. R. Donald, and D. Halperin. On the area bisectors of a polygon. Discrete and Computational Geometry, 1999. Forthcoming.

[13] O. Khatib. Real time obstacle avoidance for manipulators and mobile robots. Int. Journal of Robotics Research, 5(1):90-99, Spring 1986.

[14] E. Rimon and D. Koditschek. Exact robot navigation using artificial potential functions. IEEE Transactions on Robotics and Automation, 8(5), October 1992.

[15] K. S. J. Pister, R. Fearing, and R. Howe. A planar air levitated electrostatic actuator system. In Proc. IEEE Workshop on Micro Electro Mechanical Systems (MEMS), pages 67-71, Napa Valley, California, Feb. 1990.

[16] H. Fujita. Group work of microactuators. In International Advanced Robot Program Workshop on Micromachine Technologies and Systems, pages 24-31, Tokyo, Japan, Oct. 1993.

[17] D. Reznik and J. F. Canny. Universal part manipulation in the plane with a single horizontally vibrating plate. In P. K. Agarwal, L. Kavraki, and M. Mason, editors, Robotics: The Algorithmic Perspective. A. K. Peters, Ltd, Wellesley, MA, 1998.

[18] M. Coutinho and P. Will. Using dynamic vector force fields to manipulate parts on an intelligent motion surface. In IEEE International Symposium on Automation and Task Planning, Los Angeles, CA, 1997.

[19] D. Biegelsen, W. Jackson, A. Berlin, and P. Cheung. Air jet arrays for precision positional control of fexible media. In Int. Conf. on Micromechatronics for Information And Precision Equipment (MIPE'97), Tokyo, Japan, July 1997.

[20] J. E. Luntz and W. Messner. A distributed control system for fexible materials handling. IEEE Control Systems, 17(1), Feb. 1997.

[21] J. E. Luntz, W. Messner, and H. Choset. Parcel manipulation and dynamics with a distributed actuator array: The virtual vehicle. In Proc. IEEE Int. Conf. on Robotics and Automation (ICRA), pages 1541-1546, Albuquerque, New Mexico, Apr. 1997.

[22] K.-F. B ohringer, K. Goldberg, M. B. Cohn, R. Howe, and A. Pisano. Parallel microassembly with electrostatic force fields. In Proc. IEEE Int. Conf. on Robotics and Automation (ICRA), Leuven, Belgium, May 1998.

[23] T. L. Abell and M. Erdmann. A universal parts feeder, 1996. Personal communication / in preparation. 